\title{
The Efficacy of Pulmonary Rehabilitation Using a Mechanical In-Exsufflator and Feedback Respiratory Training for Cervical Cord Injury Patients
}

\author{
YeOnSEOP LEE, MSc, PT ${ }^{1)}$, JinSANG KIM, PhD, DVM²), Yusin Jin, MSc, PT ${ }^{1)}$ \\ 1) Major in Physical Therapy, Department of Rehabilitation Science, Graduate School, Daegu University: \\ 201, Daegudae-lo, Gyungsan-si, Kyoungbuk, 712-714 Republic of Korea. \\ TEL:+82 53-850-4653,FAX:+82 53-850-4359,E-mail: bulchun325@naver.com \\ 2) Department of Physical Therapy, College of Rehabilitation Science, Daegu University
}

\begin{abstract}
Purpose] The purpose of this research was to investigate the effect of pulmonary rehabilitation using a mechanical in-exsufflator and feedback respiratory training on cervical cord injury (SCI) patients. [Subjects and Methods] Thirty-eight patients were selected through a random sampling method and divided into two groups: an experimental group $(n=19)$ and a control group $(n=19)$. The intervention was conducted five times per week over a four-week period. [Results] In the respiratory training group, there were significance improvements in the ratio $(\% \mathrm{VC})$ of the predicted value, forced expiratory volume at one second (FEV1), and unassisted peak cough flow (UPCF) after the intervention. [Conclusion] The results suggest that pulmonary rehabilitation using a mechanical in-exsufflator and feedback respiratory training is effective at increasing the lung function, as well as augmenting the strength of cough capacity. This may reduce the respiratory system-related complications of cervical cord injury patients.
\end{abstract}

Key words: Mechanical insufflation - exsufflation (MI-E), Feedback respiratory training, SCI

(This article was submitted Jul. 14, 2011, and was accepted Sep. 12, 2011)

\section{INTRODUCTION}

Currently, the development of means of transportation and the increase in human activities have resulted in a lot of accidents and injuries, and the incidence of cervical spine injuries has also increased. The frequency of occurrence of spinal fracture and spinal damage is $55 \%$ for cervical cord damage, $35 \%$ for thoracic region damage, and $10 \%$ for lumbar region damage. A continuous increase in the number of patients with cervical cord injury has increased the demand for a reduction in cervical cord injury complications and improved quality of life ${ }^{1)}$. The representative complications of patients with cervical cord-injured patient are respiratory system complications and cardiac disorders. Respiratory system complications comprise $67 \%$ of the total, which is the highest frequency. Respiratory system complications are known to be the primary cause of death for cervical cord injury patients ${ }^{2)}$.

In patients with cervical cord injury, the damaged portion of the cervical cord may hinder the muscle functions related to breathing such as those of the accessory respiratory muscles, abdominal muscles, etc., as well as inducing paralysis in the diaphragm, expiratory muscles, and inspiratory muscles. Furthermore, this raises the possibility of ventilator distress and secretion-producing disorders within the respiratory tract. The weakening of the expiratory muscles may also cause a restrictive lung disease, which is accompanied by lung volume reduction, including lung capacity. At the same time, the weakening of inspiratory muscle strength reduces ventilation. Altogether, both types of weakening restrict periodical deep breathing, which may increase the probability of complications unless the lungs are artificially expanded.

The main reason for the occurrence of such a respiratory complication is that peak cough flow is reduced due to a lowering of the internal intra-abdominal pressure and that of the thoracic duct, which effectively eliminate secretion within the respiratory tract ${ }^{3)}$. Generally speaking, typical respiratory rehabilitation training for cervical cord injury patients includes nasal intermittent positive pressure ventilation (NIPPV), postural drainage, the chest tapping method, the vibration method, pursed lip breathing, training of expiratory and inspiratory muscles, glossopharyngeal breathing, and the abdominal push-assisted maneuver. These methods partially enhance the respiratory function and reduce the risks related to respiratory system complications.

The mechanical insufflation - exsufflation (MI-E) method alternately applies positive and negative pressure to the respiratory tract to support the effective removal of secretion from inside the respiratory system. It is also effective at increasing the peak cough flow $(\mathrm{PCF})^{4)}$. Claxton et al. found that $57 \%$ of 72 cervical cord injury patients required mechanical ventilation support ${ }^{5)}$, and that the 
management of patients' respiratory systems was considered an important factor in determining hospitalization periods and costs ${ }^{6}$. They also found that acute phase respiratory rehabilitation treatment may significantly reduce respiratory system complications ${ }^{7)}$. In the present research, we attempted to understand the effects of a non-invasive respiratory exercise conducted to increase lung ventilation using MI-E (a non-invasive respiratory exercise) in order to effectively manage lung function of spinal injury patients and to understand the effects of non-invasive respiratory exercise on the lung function in the absence of secretion in the respiratory tract. In addition, we made an effort to minimize respiratory syncytial complications by executing and utilizing concurrent treatments of invasive and non-invasive management in order to provide patients with a meaningful respiratory ability.

\section{SUBJECTS AND METHODS}

\section{Subjects}

The subjects of this research were 38 cervical cord injury patients who were hospitalized in B rehabilitation clinic located in Daejeon city between January 2010 and December 2010. Before selecting the subjects, those who had diseases related to respiration, cardiovascular problems, and orthopedic problems prior to the cervical cord injury were excluded from the subject list. The experimental method was explained to each subject, and each agreed to participate in the experiment. The subjects were assigned to the experimental and control groups according to the order of hospitalization. The general characteristics of the research subjects are given in Table 1. There were no significant differences between the degree of damage, damaged area, and the time since onset for between the experimental groups.

\section{Methods}

The methods used in this study were central nervous system movement therapies, such as joint mobilization exercise, stretching exercise, and muscle strengthening exercise. They were practiced by both the experimental group and the control group two times per day for 30 minutes, five times a week over four-week period. In addition, a forced positive pressure MI-E, along with expiratory muscle feedback respiration exercise, was practiced by the experimental group, each for 15 minutes. The forced positive pressure respiratory treatment was carried out after making the patient stand up at about 60 degrees with respect to a flat surface, and then applying positive pressure of between $20 \mathrm{cmH}_{2} \mathrm{O}$ and $40 \mathrm{cmH}_{2} \mathrm{O}$ for 1-3 seconds, and negative pressure of $-40 \mathrm{cmH}_{2} \mathrm{O}$ for $1-3$ seconds ${ }^{4}$, while avoiding hyperventilation and adjusting the strength and time according to the patient's condition ${ }^{8)}$. For the expiratory muscle feedback respiration exercise, SPIRO-BALL and TRI-BALL were used.

Lung capacity was measured using a Micro Spirometer, Micro Medical Ltd., UK, and the measured values were used by a pulmonary function calculator, Micro Medical Ltd., UK to calculate the ratio (\%VC) of predicted values, forced vital capacity (FVC), forced expiratory volume at
Table 1. Characteristics of study participants $(n=38) \quad(M \pm S E)$

\begin{tabular}{lcc}
\hline & MI-EFRTG (19) & \multicolumn{1}{c}{ CG (19) } \\
\hline Sex (male/female) & $17 / 2$ & $16 / 3$ \\
Age (years) & $45.7 \pm 3.4$ & $50.1 \pm 3.6$ \\
Height (cm) & $171.6 \pm 1.4$ & $172.1 \pm 1.2$ \\
Weight (kg) & $65.6 \pm 1.8$ & $63.7 \pm 1.8$ \\
Time since Onset (months) & $20 \pm 1.5$ & $21.4 \pm 1.2$ \\
\hline
\end{tabular}

MI-EFRTG; Mechanical Insufflation - Exsufflation Feedback Resistance training Group ; CG: Control group.

one second (FEV1), and forced expiratory ratio (FEV1/ FVC). Furthermore, a peak flow meter, Health Scan Products Inc., USA, was used to measure the unassisted peak cough flow (UPCF).

The statistically analyzed data were using SPSS 12.0 for Windows. For each group, the paired t-test was conducted to evaluate the effectiveness verification of the treatment, and the independent t-test was carried out to compare the change in respiratory function between the two groups. The level of significance was chosen as 0.05 .

\section{RESULTS}

There were no significant differences between the general characteristics of the two groups. In the comparison of the values of respiratory function before and after the respiratory rehabilitation treatment, the experimental group showed a significant increase in $\mathrm{VC} \%(\%), \mathrm{FEV} 1$ (L), and UPCF (L/min); FEV1/FVC (\%) showed an increase but it was not significant (Table 2).

In the comparison of changes in respiratory function after the respiratory rehabilitation treatment between the experimental and control group, there were significant differences between the changes in VC\% (\%), FEV1 (L), and UPCF (L/min). The change in FEV1/FVC (\%) was higher in the experimental group, but without significance (Table 3).

\section{DISCUSSION}

Patients with cervical cord injuries experience paralysis in the expiratory and inspiratory muscles, which results in pulmonary disabilities. These, in turn, raise the risk of respiratory syncytial complications such as atelectasis and pneumonia. Therefore, the regular evaluation and effective maintenance of such respiratory syncytial complications are essential to decrease the prevalence of such complications and the death rate of patients with cervical cord injuries, while improving their life quality ${ }^{9}$.

The main reasons for the prevalence of respiratory syncytial complications in patients with cervical cord injuries are the difficulty in excreting airway internal secretion, because of weakened respiratory muscles, and the lack of production of peak cough flow. The weakening of respiratory muscles correlates with the degree of damage and the abnormal control of nerves in the various damaged areas; damage in the upper cervical cord (C1-C4) increases the prevalence rate ${ }^{10)}$. Bach reported that peak cough flow 
Table 2. Comparison of Pulmonary Function Data between the Control and Experimental Groups $(\mathrm{M} \pm \mathrm{SE})$

\begin{tabular}{lccccc}
\hline \multirow{2}{*}{ Pulmonary Function Data } & \multicolumn{2}{c}{ CG } & & \multicolumn{2}{c}{ MI-EFRTG } \\
\cline { 2 - 3 } \cline { 5 - 6 } & Preintervention & Postintervention & & Preintervention & Postintervention \\
\hline VC\% (\%) & $40.1 \pm 5.7$ & $41.3 \pm 5.6$ & & $42.3 \pm 4.9$ & $47.0 \pm 4.7^{*}$ \\
FEV1 (L) & $1.3 \pm 0.1$ & $1.3 \pm 0.1$ & & $1.3 \pm 1.1$ & $1.5 \pm 0.1^{*}$ \\
FEV1/FVC (\%) & $69.6 \pm 3.9$ & $71.2 \pm 3.8$ & & $73.5 \pm 3.5$ & $74.4 \pm 2.6$ \\
UPCF (L/min) & $158.9 \pm 38.5$ & $165.2 \pm 38.6$ & & $153.4 \pm 29.0$ & $188.1 \pm 30.1^{*}$ \\
\hline
\end{tabular}

Value are Mean $\pm \mathrm{SE} ; *=\mathrm{p}<0.05$. VC\%: vital capacity ratio ; FVC: forced vital capacity; FEV1: forced expiratory volume at one second; FEV1/FV : forced expiratory ratio; UPCF: unassisted peak cough flow.

may be enhanced by increasing maximum insufflation capacity ${ }^{11)}$. Accordingly, we simultaneously conducted MI-E and exhalation feedback respiratory exercises to the effects of cervical cord injury on the pulmonary function and cough abilities and to promote effective patient maintenance. The lung capacity of the pulmonary function and respiratory function were measured using a Micro Spirometer (Micro Medical Ltd, UK), while cough ability was determined using a Peak Flow Meter (Cardinal Health 232 Ltd, UK). Forced volume vital capacity and forced exhalation capacity for 1 second serve as important indicators of respiratory obstruction.

In a study providing chronic/incurable obstructive pulmonary disease patients with respiratory rehabilitation training, DiMarco et al. a method of producing an effective $\operatorname{cough}^{12)}$ and Kim's patients' respiratory treatment demonstrated a relative increase in the peak cough flow ${ }^{13}$. MI-E was employed in research by Chang-il Park et al. They showed a relative increase in the maximum insufflation capacity and non-conservative peak cough flow during respirator rehabilitation training. All of these findings are in agreement with the results of the present research ${ }^{4}$. In contrast, Kim and Ries report that respiratory obstruction treatment was not effective, as different degrees of respiratory obstruction showed different treatment results ${ }^{14,15}$ ) .However, their finding were due to decreases in the lung capacity, inspiratory capacity, and functional residual volume of patients with cervical cord injury which resulted in reduced thoracic elasticity.

Brach, using MI-E, reported the recovery of patients with respiratory and nervous system infections ${ }^{16)}$. Bach reported increases in the peak flow and non-conservative peak cough flow, and reported positive results for neuromuscular disease patients ${ }^{17)}$. The results were explained by the effective removal of airway secretion, as MI-E treatment generates higher peak flow cough than other methods.

Cervical cord impairments generally induce paralysis and stiffening of the respiratory and intercostal muscles, and decrease the elasticity of the lung and chest by limiting the movement of the iliocostal muscles. ${ }^{18)}$ The reports of Kang and Bach note the importance of an increase in lung capacity $^{19)}$.

The results of the present study indicate that forced lung capacity was increased. The experimental group's increase in respiratory function, due to consistent insufflation effects, effectively improved the movement of the lung and chest, as well as the lung's elasticity, and this provides meaningful
Table 3. Comparison of the Changes in Pulmonary Function Data between the Control and Experimental Groups after Treatment $(\mathrm{M} \pm \mathrm{SE})$

\begin{tabular}{llc}
\hline Pulmonary Function Data & CG & MI-EFRTG \\
\hline VC\% (\%) & $1.1 \pm 0.7$ & $4.7 \pm 1.1^{*}$ \\
FEV1 (L) & $0.2 \pm 0.05$ & $0.1 \pm 0.0^{*}$ \\
FEV1/FVC (\%) & $1.1 \pm 4.6$ & $0.8 \pm 2.9$ \\
UPCF (L/min) & $6.3 \pm 4.9$ & $34.7 \pm 7.6^{*}$ \\
\hline
\end{tabular}

$*=\mathrm{p}<0.05$

evidence of improved peak cough flow. Thus, diverse treatment methods must be attempted in cases of cervical cord injury.

In conclusion, in this study, 38 patients with cervical cord injuries performed exercises for four weeks, and we assessed how MI-E and expiratory training affected their lung and cough capacities. In respiratory training group, the ratio $(\% \mathrm{VC})$ of the predicted value, forced expiratory volume at one second (FEV1), and unassisted peak cough flow (UPCF) showed significant differences between pre-and post-intervention, and in the comparison between the experimental and control groups, the changing in the ratio $(\% \mathrm{VC})$ of the predicted value, FEV1, and UPCF were significantly greater in the experimental group, demonstrating that MI-E and inspiratory feedback training enhance lung and cough capacities which may reduce the respiratory syncytial complications of patients with cervical cord injuries.

\section{REFERENCES}

1) Widerstrom-Noga EG, Felipe-Cuervo E, Broton JG, et al.: Perceived difficulty in dealing with consequences of spinal injury. Arch Phys Med Rehabil, 1999, 80: 580-586.

2) BEC: Respiratory rehabilitation for spinal cord injury patient. http://breatheasyclub.com (Accessed Nov. 25, 2007).

3) Cotton BA, Pryor JP, Chinwalla I, et al.: Respiratory complications and mortaliry risk associated with thoracic spine injury. Journal of TraumaInjury Infection \& Critical Care, 2005, 59: 1400-1409.

4) Park CI, Shin GC, Kang SW, et al.: The efficacy of pulmonary rehabilitation using mechanical in-exsufflator in cervical cord injured patients. The Journal of Korean Academy of Rehabilitation, 2002, 26: 403-408.

5) Claxton R, Wong D, Cung F, et al.: Predictors of hospital mortality and mechanical ventilation in patients with cervical spinal injury. Can J Anaesth, 1998, 45: 144-149.

6) Winslow C, Bode RK, Felton D, et al.: Impact of respiratory complication 
on length of stay and hospital costs in acute cervical spinal injury. Chest, 2002, 121: 1548-1554.

7) Berlly $M$, Shem K: Respiratory management during the first five days after spinal cord injury. J Spinal Cord Med, 2007, 30: 309-318.

8) Liaw MY, Lin MC, Cheng PT, et al.: Resistive inspiratory muscle training: its effectivenss in patients with acute complete cervical cord injury. Arch Phys Med Rehabil, 2000, 81: 752-756.

9) Baydur A, Adkins RH, Milic-Emili J, et al.: Lung mechanics in individual with spinal cord injury: effects of injury level and posture. J Appl Physiol, 2001, 90: 405-411.

10) Jackson $\mathrm{AB}$, Groomed TE: Incidence of respiratory complications following spinal cord injury. Arch Phys Med Rehabil, 1994, 75: 270-275.

11) Bach JR, Smith WH, Michaels J, et al.: Airway secretion clearance by mechanical exsufflation for post-poliomyelitis ventilator-assisted individuals. Archives of Physical Medicine and Rehabilitation, 1993, 74: $170-177$.

12) DiMarco AF, Kowalski KE, Geertman RT, et al.: Spinal cord stimulation: A new method to produce an effective cough in patients with spinal cord injury. Am J Resp \& Criti Care Med, 2006, 173: 1386-1398.
13) Kim EK, Lee BS, Kim DA, et al.: Effect of 4 weeks respiratory rehabilitation program for hospitalized cervical spinal cord injury patients. Korean J Rehabil Nurs, 2007, 10: 108-115.

14) Kim AK: The study on the effects of a respiratory rehabilitation program for COPD patients. J Korean Acad Nurs, 2001, 31: 257-277.

15) Ries AF, Kaplan EM, Limburg TM, et al.: Effects of pulmonary rehabilitation on physiological and phychological outcomes in patients with chronic obstructive pulmonary disease. Annal of Inter Med, 1995, 122: 823-832.

16) Barach AL, Beck GJ: Exsufflation with negative pressure: physiologic and clinical studies in poliomyelitis, bronchial asthma, pulmonary emphysema and bronchiectasis. Arch Intern Med, 1954, 43: 549-566.

17) Bach JR: Inappropriate weaning and late onset ventilatory failure of individuals with traumatic spinal cord injury. Paraplegia, 1993, 31: 430-438.

18) Estenne M, DeTroyer A: The effects of tetraplegia on chest wall statics. Am Rev Respir Dis, 1986, 134: 121-124.

19) Kang SW, Bach JR: Maximum insufflation capacity. Chest, 2000, 118: $61-65$. 